\title{
Feasibility of Low-Dose 68Ga-DOTATATE With Short Acquisition Time Using Total-Body PET/CT in Patients With Neuroendocrine Tumor
}

Jie Xiao

Zhongshan Hospital Fudan University

Haojun Yu

Zhongshan Hospital Fudan University

Hongyan Yin

Zhongshan Hospital Fudan University

Guobin Liu

Zhongshan Hospital Fudan University

Yan $\mathrm{Hu}$

Zhongshan Hospital Fudan University

Lin Qinyu

Zhongshan Hospital Fudan University

Zhao Yanzhao

Zhongshan Hospital Fudan University

Yiqiu Zhang

Zhongshan Hospital Fudan University

Pengcheng Hu

Zhongshan Hospital Fudan University

Dengfeng Cheng

Zhongshan Hospital Fudan University

Hongcheng Shi ( $\nabla$ shi.hongcheng@zs-hospital.sh.cn )

Zhongshan Hospital Fudan University

\section{Research Article}

Keywords: Image quality, low-dose regimen, total-body PET, 68Ga-DOTATATE, PET/CT imaging

Posted Date: July 7th, 2021

DOI: https://doi.org/10.21203/rs.3.rs-667845/v1 
License: (c) (i) This work is licensed under a Creative Commons Attribution 4.0 International License. Read Full License 
Feasibility of low-dose ${ }^{68}$ Ga-DOTATATE with short acquisition time using total-body PET/CT in patients with neuroendocrine tumor

Jie Xiao ${ }^{1,2,3 \#}$, Haojun $\mathrm{Yu}^{1,2,3 \#}$, Hongyan Yin ${ }^{1,2,3}$, Guobin $\mathrm{Liu}^{1,2,3}$, Yan $\mathrm{Hu}^{1,2,3}$, Lin Qinyu ${ }^{1,2,3}$, Zhao Yanzhao ${ }^{1,2,3}$, Yiqiu Zhang $^{1,2,3,4}$, Pengcheng Hu${ }^{1,2,3}$, Dengfeng Cheng ${ }^{1,2,3^{*}}$, Hongcheng Shi ${ }^{1,2,3,4^{*}}$

${ }^{1}$ Department of Nuclear Medicine, Zhongshan Hospital, Fudan University, 180 Fenglin Road, Shanghai, 200032, China.

${ }^{2}$ Institute of Nuclear Medicine, Fudan University, Shanghai, 200032, China.

${ }^{3}$ Shanghai Institute of Medical Imaging, Shanghai, 200032, China.

${ }^{4}$ Collaborative Innovation Center for Molecular Imaging Precision Medicine, Shanxi Medical University, Taiyuan, Shanxi, 030001, People’s Republic of China.

\#These authors contributed equally to this article.

* Corresponding to: Dengfeng Cheng and Hongcheng Shi,

E-mail address: Cheng.dengfeng@zs-hospital.sh.cn or Shi.hongcheng@zs-hospital.sh.cn

Telephone: +8613681971579, Fax: +86-21-62489191.

Department of Nuclear Medicine, Zhongshan Hospital, Fudan University;

No. 180 in Fenglin Road, Shanghai 200032, P.R. China. 


\section{Abstract}

Purpose To explore the feasibility of a low dose regimen with short acquisition time of ${ }^{68}$ Ga-DOTATATE total-body PET/CT without compromising image quality of patients with NETs.

Methods Fifty-seven consecutive NETs patients who underwent ${ }^{68}$ Ga-DOTATATE total-body PET/CT, with a low dose regimen (0.8-1.2 MBq/kg) of ${ }^{68} \mathrm{Ga}$-DOTATATE and acquisition time of $10 \mathrm{~min}$ prior to any treatment, were enrolled in the present study. The PET data were split into $1 \mathrm{~min}, 2 \mathrm{~min}, 3 \mathrm{~min}, 4 \mathrm{~min}, 5 \mathrm{~min}, 8 \mathrm{~min}$ and $10 \mathrm{~min}$ reconstruction groups, referenced as R1, R2, R3, R4, R5, R8 and R10. The subjective evaluation of image quality was scored in 5-point Likert scale based on three aspects: the overall impression of the image quality, the image noise, the lesion detectability. The objective image quality was assessed by the signal-to-noise ratio of liver $\left(\mathrm{SNR}_{\mathrm{L}}\right)$, the coefficient of variation $(\mathrm{CV})$, the SUVmax, SUVmean, SD of liver, mediastinal blood pool and lesion, the tumor-liver ratio (TLR), the tumor-mediastinal blood pool-ratio (TMR) of lesion.

Results The sufficient subjective image quality with a score of $3.44 \pm 0.53$ could be obtained at 3 min acquisition duration, with a kappa value of 0.90 . In quantitative analysis, the value of $\mathrm{SNR}_{\mathrm{L}}$ is over 10 in all reconstruction groups. As the acquisition time increases, $\mathrm{SNR}_{\mathrm{L}}$ was increased and $\mathrm{CV}$ was decreased within 3 min, while $\mathrm{SNR}_{\mathrm{L}}$ and $\mathrm{CV}$ showed no significant different between R4-R10. There was no significant different in TMR and TLR of lesion between R1-R10 (all $p<0.05)$. Referenced as PET images of R10, 90 SSTR-positive lesions are identified, and all those lesions are found in the R1-R10 groups (100\%).

Conclusion The low-dose (0.8-1.2 MBq/kg) ${ }^{68} \mathrm{Ga}$-DOTATATE total-body PET/CT not only shortens acquisition time, but maintains a sufficient image quality for the NETs patients.

Keywords Image quality, low-dose regimen, total-body PET, ${ }^{68}$ Ga-DOTATATE, PET/CT imaging 
Neuroendocrine tumors (NETs) are a heterogeneous group of tumors originating from neuroendocrine cells distributed in many organs, but more in the gastrointestinal tract and lungs, synthesizing, storing and secret various circulating hormones and neurotransmitters [1]. NETs are marked by the expression of somatostatin receptors (SSTR) subtypes (SSTR1-SSTR5), among which SSTR2 is predominately expressed [2]. In well-differentiated NETs (G1, G2 and well-differentiated G3), the high SSTRs expression not only makes these lesions ideals of targeting radionuclide imaging, but also indicates the potentially effective for radionuclide therapy of NETs [3]. Scintigraphy with ${ }^{123}$ I-somatostaion analogues, and followed by ${ }^{111} \mathrm{In},{ }^{99 \mathrm{~m} T c}$ labelling, using planar, SPECT or SPECT/CT imaging, have been effectively used for targeting SSTRs imaging. However, it has several limitations, including high physiological uptake in liver, spleen and kidney, etc., high energy gamma emissions and lower resolution of gamma cameras, all resulting in decreasing the detection efficiency [4, 5].

Recently, PET imaging with ${ }^{68} \mathrm{Ga}$ - DOTATATE $\left({ }^{68} \mathrm{Ga}\right.$ - DOTA- Tyr3- Thr8- octreotide), showed especially higheraffinity in binding to SSTR2-positive tissues $(0.2 \pm 0.04 \mathrm{nM})$ than that of the other SSTR subtypes $[3,6]$. Better spatial resolution, higher sensitivity and shorter acquisition times were achieved dependent on the revolution of PET detector. The current procedure guideline on ${ }^{68} \mathrm{Ga}$-DOTA-conjugated peptides recommended the administered activity ranging from 100 to $200 \mathrm{MBq}$, and at least $100 \mathrm{MBq}$ was needed in order to obtain a good image quality. The amount of DOTA-peptide should be less than $50 \mu \mathrm{g}$ for no significant pharmacological effect. Additionally, the injection activity was also limited by the sensitivity of PET detector and patient's weight [7]. Nowadays, the total-body PET detector crystals with size of $2.76 \times 2.76 \times 18.0 \mathrm{~mm}^{3}$, coupled to silicon photomultiplier (SIPMs), owned ultra-high sensitivity [8]. Based on this feature, our team found that low injected ${ }^{18} \mathrm{~F}-\mathrm{FDG}$ activity was sufficient for a good image quality and without decreasing the detection efficiency for lung cancer $[9,10]$. And ultra-low dose $(0.37 \mathrm{MBq} / \mathrm{kg})$ injected activity of ${ }^{18} \mathrm{~F}$-FDG could achieve relevant kinetic metrics and comparable image quality compared with that of full-activity imaging [11]. Besides, using conventional dose-regimen, the acquisition time could be significantly shorter within 2 min for a sufficient image quality of ${ }^{18}$ F-FDG PET/CT [12]. 
By virtue of the ultra-high sensitivity of total-body PET detectors, we initially explore the feasibility of a low injected dose regimen (0.8-1.2 MBq/kg) with short acquisition time of ${ }^{68} \mathrm{Ga}$-DOTATATE total-body PET/CT, and without compromising image quality of patients with NETs.

\section{Materials and Methods}

\section{Patient population}

All patients' information was in accordance with the institutional ethical standards, and all included patients waived written informed consent prior to recruiting into the study. A total of 102 consecutive patients diagnosed or suspected neuroendocrine tumors (NETs) underwent ${ }^{68} \mathrm{Ga}$-DOTATATE total-body PET/CT from September 2020 to January 2021 in our center. Fig. 1 showed the process of patient recruitment including the inclusion and exclusion criteria. Finally, 57 patients had histologically confirmed NETs (46 of all included patients received surgical or endoscope resection, and 11 underwent aspiration biopsy), and then graded according to AJCC 2017 [14]. ${ }^{68}$ Ga-DOTATATE performed for diagnosis, staging or restaging. All included patients were grouped into SSTR positive expression group and negative expression group.

\section{${ }^{68}$ Ga-DOTATATE PET/CT imaging and image reconstruction}

${ }^{68} \mathrm{Ga}$-DOTATATE were synthesized in-house according to the method previously described by Zhernosekov [15]. All participants had no need for fasting food before the injection. The imaging was acquired as following steps, shown in Fig. 2: (1) Low radiation dose CT was performed prior to PET imaging for attenuation correction with voltage of $120 \mathrm{kV}$, the current of $10 \mathrm{~mA}$. (2) The images were simultaneously acquired in a dynamic mode for 60 min with a bolus injection of ${ }^{68} \mathrm{Ga}$-DOTATATE by uEXPLORER (United Imaging Healthcare, Shanghai, China) with an axial FOV of $194 \mathrm{~cm}$. (3) Then, the tube voltage of Diagnostic CT was performed with the voltage of $120 \mathrm{kV}$, and the modulation technology was recommended for tube current to reduce the radiation dose. (4) The 60 min images were divided into two parts: 0-50 min and 50-60 min, and the images of 50-60 min were further split into 50-51 min, 50-52 $\mathrm{min}, 50-53 \mathrm{~min}, 50-54 \mathrm{~min}, 50-55$ 
min, 50-58 min and 50-60 min reconstruction groups, which were defined as R1, R2, R3, R4, R5, R8 and R10. The injection activity was in a linear dose regimen $(0.8-1.2 \mathrm{MBq} / \mathrm{kg})$.

Table 1 summarized the current scanning and reconstruction protocol. PET images were reconstructed using 3D list-mode ordered-subset expectation maximization algorithm (3D-OSEM), combination with following parameters: Time of fly and point spread function modeling (TOP-PSF), 3 iterations with 20 subsets, matrix of $192 \times 192$ and slice thickness of 1.443 $\mathrm{mm}$, and the full width at half maximum of the Gaussian filter function of $3 \mathrm{~mm}$.

\section{Image analysis}

PET image quality was independently evaluated by two experienced nuclear medicine physicians, who were blinded to the patient's medical history and the reconstruction time. For subjective assessment of image quality, the image quality was scored in 3 perspectives: the overall impression of the image quality, the image noise, the lesion detectability, which were scored using 5-points Likert scale (1, non-diagnostic image quality; 2, poor image quality; 3, moderate image quality; 4, good image quality; 5, excellent image quality), as previously described [10]. Images from R10 were regarded as excellent in the analysis. The score of 3 was equivalent to the image quality of a clinical routine in our center.

Then, the image was interpreted by an experienced nuclear medicine physician. A circular region of interest (ROI) in 2D mode was manually drawn in the right liver lobe and the ascending aorta in a lesion-free, homogenous region, avoiding partial volume effects. The diameter of the ROI in the right liver lobe was limited to $20 \mathrm{~mm}$, and the perimeter of ROI in the ascending aorta was $30 \mathrm{~mm}^{2}$. The ROI of lesion was placed in the highest-pixel-value slice of transverse view, and the highest lesion was measured in case of multiple lesions. The maximum standard uptake value (SUVmax), the mean of standard uptake value (SUVmean), and the standard deviation (SD) of liver and ascending aorta were recorded. The SUVmax and SUVmean of a lesion were recorded. For interpretation of image quality, the signal-to-noise ratio $\left(\mathrm{SNR}_{\mathrm{L}}\right)$ of liver was calculated by dividing the SUVmean by SD:

$$
\mathrm{SNR}_{\mathrm{L}}=\frac{\text { SUVmean of liver }}{\mathrm{SD} \text { of liver }}
$$


The noise can influence the detectability of lesion, described as the coefficient of variation (CV):

$$
\mathrm{CV}=\frac{\mathrm{SD}}{\text { SUVmean }} \quad \times 100 \%
$$

2

ROI was first placed on R10 images, and then the measurement of the same location on the other reconstruction images

using the copy-and-paste ROI function. The SUVmax, SUVmean and SD were recorded, respectively. The tumor-liver ratio (TLR) and tumor-mediastinal blood pool-ratio (TMR) was calculated by dividing the SUVmax of the lesion by the SUVmean of liver, and SUVmax of the lesion by the SUVmean of ascending aorta:

$$
\begin{aligned}
& \mathrm{TLR}=\frac{\text { SUVmax of lesion }}{\text { SUVmean of liver }} \\
& \mathrm{TMR}=\frac{\text { SUVmax of lesion }}{\text { SUVmean of ascending aorta }}
\end{aligned}
$$

Lesions' detectability was measured by the lesion detection rate.

\section{Statistical analysis}

All statistical analysis was performed using IBM SPSS Statistics Version 26 (IBM Inc., Chicago, IL, USA) and Prism 8 (GraphPad Software Inc., San Diego, California, USA). Data was described as mean \pm SD. Differences in quantitative variables were assessed by analysis of variance (ANOVA) with post hoc Bonferroni adjustment for pairwise comparison. Categorical variables were compared using the chi square test. Cohen's kappa analysis of overall image quality was performed to evaluate the inter-reader agreement. Results were considered statistically significant if p less than 0.05 .

\section{Results}

\section{Patient characteristics}

The patients' characteristics are displayed in Table 2. A total of 57 NET patients (36 males and 21 females; mean age, 53.6 \pm 11.2 years, range: $30.0-80.0$ years) were enrolled in the study. The injected activity per kilogram was $1.0 \pm 0.2$ (range: $0.8-1.2 \mathrm{MBq} / \mathrm{kg}$ ), and the mean injected activity was $66.9 \pm 11.3 \mathrm{MBq}$ (range: $41.9-88.0 \mathrm{MBq}$ ). The images showed the lesions were located in the gastrointestinal $(39,68.4 \%)$, pancreas $(5,8.8 \%)$, liver $(4,7.0 \%)$, breast $(2$, 
$3.5 \%)$, mediastinum $(2,3.5 \%)$ and another site $(5,8.8 \%)$. According to AJCC classification, there are 18 patients with G1 (31.6\%), 29 patients with G2 (36.8\%) and 10 patients with G3 or neuroendocrine carcinoma (17.5\%).

\section{Analysis of image quality}

\section{Subjective image quality}

Table 3 gives an overview of subjective score for each reconstruction, the score of overall image quality in R1 and $\mathrm{R} 2$ group were $1.51 \pm 0.50$ and $2.44 \pm 0.54$, reviewed as non-diagnostic image. The R3-R10 presents a good image quality with the score of $3.44 \pm 0.53,4.44 \pm 0.57,5.00 \pm 0.00,5.00 \pm 0.00$ and $5.00 \pm 0.00$, respectively. The inter-reader agreement of overall image quality in the R1-R10 groups indicated almost perfect with a kappa value of $0.93,0.86$, 0.90 and $0.97,1.00,1.00,1.00$ for each acquisition duration, respectively. The noise in R1 and R2 groups did not meet the requirement of diagnosis with the subjective score of $1.44 \pm 0.50$ and $2.40 \pm 0.53$, while the noise score of the R3-R10 showed superior to R1 and R2. The level of noise in the R3-R10 is scored with $3.42 \pm 0.60,4.47 \pm 0.50,5.0 \pm 0.0$, $5.0 \pm 0.0$ and $5.0 \pm 0.0$, respectively. The subjective score of lesion detectability were $2.59 \pm 0.62,3.56 \pm 0.67,4.47 \pm 0.57$, $4.97 \pm 0.18,5.0 \pm 0.0,5.00 \pm 0.00$ and $5.00 \pm 0.00$ for all those reconstruction groups.

\section{Objective image quality}

The value of $\mathrm{SNR}_{\mathrm{L}}$ is over 10 in all reconstruction groups. In Fig. 3a, the $\mathrm{SNR}_{\mathrm{L}}$ were significantly lower in R1, R2 and R3 than R10 group, while R4, R5, R8 and R10 show no significant differences ( $p<0.05$ ). The CV is presented against acquisition time in Fig. 3b, and the CV is significantly decreased along with the reconstruction times in R1, R2, R3 compared with R10, but it is no obvious difference between R4, R5, R8 and R10. There is no significant different in TMR and TLR between all reconstruction groups (all $p<0.05$, shown in Fig. 3c-d). No differences in SUVmax and SUVmean of liver are evident between different groups. The SD of the liver is significantly higher in the R1 group compared with the R8 and the R10 group, and the equal value of SD is observed between the other groups (Shown in Fig. 4a). There are no obvious differences in SUVmax and SUVmean of mediastinal blood pool, 
and the SD of mediastinal blood pool in R1, R2 and R3 are higher than R4-R10 (shown in Fig. 4b). There is not any discrepancy between a series of reconstructions in SUVmax, SUVmean and SD of lesions (shown in Fig. 4c). Table 4 presented objective image quality parameters in R4 and R10 group. Compared with R10, R4 obtain equal value of SUVmax and SUVmean in liver, blood pool and lesions. And the CV of liver and blood pool also shows no significant differences. The TLR and TMR in R2 are comparable to that of R10.

Referenced as PET images of R10, 90 SSTR-positive lesions are identified. There are in liver $(38,42.2 \%)$, bone (18, $20 \%)$, lymph node $(15,16.7 \%)$, gastrointestinal $(10,11.1 \%)$, pancreas $(5,5.6 \%)$, breast $(2,2.2 \%)$ and mediastinum (2, 2.2\%), and all those lesions are found in the R1-R10 groups (100\%). Fig. 5 showed a typical case.

\section{Discussion}

This study investigated the objective image quality, subjective image quality and lesion detectability to search for an optimize and minimize acquisition time of low-dose ${ }^{68} \mathrm{Ga}$-DOTATATE (0.8-1.2 MBq/kg) PET/CT imaging. The results demonstrated that sufficient subjective image quality with a score of $3.44 \pm 0.53$ could be obtained at 3 min acquisition duration, and the lesions acceptable for diagnosis at duration time of 2 min with a score of $3.56 \pm 0.67$. The quantitative parameters of image quality in acquisition time of 4 min were comparable to that of $10 \mathrm{~min}$.

There are multiple factors can influence image quality of PET of ${ }^{68} \mathrm{Ga}$-DOTATATE, such as treatment with somatostatin analogs, injected activity, acquisition time, uptake time, presence of extravasation, uptake time, the sensitivity of PET detector system, reconstruction parameters receptor density, etc. To minimize the influence of affected factors as soon as possible on image quality, all participants included in this study were following the standard procedures established by our center. Previous studies found that ${ }^{68} \mathrm{Ga}$-DOTATATE had less uptake in liver, spleen, and thyroid after treatment initiation with somatostatin analogs than patients without treatment, but no significant differences were found in the uptake of ${ }^{68}$ Ga-DOTATATE in lesions [16]. One prospective study performed ${ }^{68}$ Ga-DOTATATE imaging 1 day before and 1 day after injection of lanreotide, and no evidence of 
decreasing uptake in tumor, but higher tumor-to-liver ratio was obtained [17]. To avoid the influence of treatment, our study excluded 6 patients with continuous treatment with octreotide and 1 patient with high intensity focused ultrasound in liver within 1 week.

PET image quality also depends on the acquisition time and the amount of administered activity. Administered activity of ${ }^{68} \mathrm{Ga}$-DOTATATE should be determined by Diagnostic Reference Levels (DRLs), which do not exceed standard procedures when good and normal practice is applied. The current procedure guidelines recommended the activity administered should be over $100 \mathrm{MBq}$ for a good image quality for consideration of the sensitivity of PET detector and patient's weight. Based on the features of ultra-high sensitivity of total-body PET detector crystals, it is possible for images of diagnostic quality with low injection activity and a minimum acquisition time. Compared with recommended activity, the total injected activity in this study was at a relatively lower level $(0.8-1.2 \mathrm{MBq} / \mathrm{kg})$, ranging from 41.9 to $88.0 \mathrm{MBq}$ for the body weight in the range of $42.8 \mathrm{of} 101.8 \mathrm{~kg}$. The biodistribution and radiation dosimetry showed that ${ }^{68} \mathrm{Ga}$-DOTATATE was the highest uptake in spleen, followed by bladder and liver, and the total effective dose was $0.021 \pm 0.003 \mathrm{mSv} / \mathrm{MBq}$ [13]. Thus, the radiation dose to patients could be markedly reduced, which is consistent with the recommended radiation principle of as low as reasonably possible (ALARP).

Longer acquisition time could to some extent compensate for the decrease in total count of low-dose regimen, increase the $\mathrm{SNR}_{\mathrm{L}}$, and reduce image noise $(\mathrm{CV})$, making the reduction of SD in liver and blood pool. However, longer scan time also reduced patient's tolerance and increased the possibility of motion during the examination. In this study, the subjective analysis found that 3 min acquisition duration could meet the clinical need of diagnosis. The objective parameters were no significant difference between 4 and $10 \mathrm{~min}$, and the lesions could be clearly detected in all acquisition time groups (100\%). Compared with 3-5 beds acquisition time of 3 min per bed and 30\% overlap, the duration of single bed of total-body with 3-4 min could be obviously shortened without compromising the image quality. 
The $\mathrm{SNR}_{\mathrm{L}}$ method might also be affected by variation in liver uptake and ${ }^{68} \mathrm{Ga}$-DOTATATE shows high concentration in liver, which could not provide a complete measure of image quality and yield adequate information about image resolution and contrast recovery. However, the $\mathrm{SNR}_{\mathrm{L}}$ is relatively easy to calculate, and correlations with human observer studies. Thus, $\mathrm{SNR}_{\mathrm{L}}$ was always an optimal parameter to be chosen [18]. In Fig. 3a, the average $\mathrm{SNR}_{\mathrm{L}}$ over 10 through all duration time groups, which fulfill the Rose criterion: $\mathrm{SNR}_{\mathrm{L}}$ needs to be equal to or over than 5. The higher $\mathrm{SNR}_{\mathrm{L}}$ is expected to lower noise level. The CV varies from body weight, and overweight patients were significantly larger than that of normal weight. Nageki et al [19] reported that the CVliver of PET images for patients weighted 60-74 $\mathrm{kg}$ was $10.98 \%$, and that for the patient weighted over $75 \mathrm{~kg}$ was $14.15 \%$. A recent study [20] showed that 5.5 times noise reduction of a 194-cm FOV PET compared to a $30-\mathrm{cm}$ FOV digital PET with the same total examination time for scanning a $2-\mathrm{m}$-long phantom, and the noise reduction became 1.5 times when the same acquisition time per bed was performed. In Fig. 3b, the average CVliver of injection activity of $0.8-1.2 \mathrm{MBq} / \mathrm{kg}$ below $10 \%$ with body weight ranging from $42.8 \mathrm{~kg}$ to $101.8 \mathrm{~kg}$ was obtained in all acquisition groups, and the CVliver in 4 min was comparable to $10 \mathrm{~min}$.

In addition, the image quality could also be improved using the reconstructed method of PSF and TOF. Previous study [21] reported that the TOF could accelerate the vergence for a $13 \mathrm{~mm}$ sphere and obtain more contact information than that without TOF information. Although the image reconstructed with PSF correction slowed the iterative convergence, it could provide a more uniform background and increased $\mathrm{SNR}_{\mathrm{L}}$ than that without PSF correction. Previous studies showed that a combination of PSF and TOF clearly improved image quality [22]. In this study, the low-dose regimen of ${ }^{68} \mathrm{Ga}$-DOTATATE imaging with shorter acquisition time, sufficient image quality and high lesions detectability, which might also be contributed by the combination of ultra-high sensitivity of total body detector and the image reconstructed by TOF and PSF.

There are several limitations in our study. Firstly, the dose-regimen was only based on body weight. Although we 
had a relatively large range of body weight, the influence of patient sized on image quality was not detailly investigated. Secondly, the results were only based on total-body PET detector, whether the optimal and minimized time of whole-body PET scanner in the same condition was equal to or longer than 4 min needed to be further investigated. Third, the sample size of SSTR-positive patients was limited in our study. Thus, further investigation should be performed to evaluate the influence of stages of NETs on lesion detectability.

\section{Conclusion}

The low-dose (0.8-1.2 MBq/kg) ${ }^{68} \mathrm{Ga}$-DOTATATE total-body PET/CT not only shortens acquisition time, but maintains a sufficient image quality for the NETs patients.

\section{Funding}

This study is supported by the Three-year Action Plan of Clinical Skills and Innovation of Shanghai Hospital Development Center (Grant number: SHDC2020CR3079B), and Science and Technology Committee of Shanghai Municipality (Grant number: 20DZ2201800), Special Fund for Clinical Research, Young Program of Zhongshan Hospital of Fudan University (Grant number: 2019ZSYQ28, 2020ZSLC63), Young Program of Zhongshan Hospital and Shanghai "Rising Stars of Medical Talent"-Youth Development Program (Grant number: HWJRS2019-720), the Shanghai Municipal Key Clinical Specialty Project (Grant number: SHSLCZDZK03401). Next generation information infrastructure construction project from Shanghai Municipal Commission of Economy and Informatization (Grant number: 201901014).

\section{Ethical approval}

All procedures performed in studies involving human participants were in accordance with the ethical standards of the institutional and/or national research committee and with the 1964 Helsinki declaration and its later amendments 
or comparable ethical standards.

\section{Authors' Contributions}

Jie Xiao was involved in the statistical analysis and manuscript writing. Haojun Yu contributed to data acquisition and reconstructions. Guoling Liu and Hongyan Yin contributed to data analyses and image interpretation. Yan Hu helped with data processing. Yiqiu Zhang and Pengcheng Hu supervised the study. Qingyu Lin and Yanzhao Zhao were responsible for pharmaceuticals' synthesis and quality control. Hongcheng Shi and Dengfeng Cheng designed the study and contributed to editing and reviewing the manuscript. All authors read and approved the final manuscript.

\section{Conflicts of interest}

We declare that all authors have no conflicts of interest. 


\section{References}

1. Dasari A, Shen C, Halperin D, Zhao B, Zhou S, Xu Y, et al. Trends in the incidence, prevalence, and survival outcomes in patients with neuroendocrine tumors in the United States. JAMA Oncol. 2017;3(10):1335-42.

2. Reubi JC, Waser B. Concomitant expression of several peptide receptors in neuroendocrine tumours: molecular basis for in vivo multireceptor tumour targeting. Eur J Nucl Med Mol Imaging. 2003;30(5):78193.

3. Reubi JC, Schär JC, Waser B, Wenger S, Heppeler A, Schmitt JS, et al. Affinity profiles for human somatostatin receptor subtypes SST1-SST5 of somatostatin radiotracers selected for scintigraphic and radiotherapeutic use. Eur J Nucl Med Mol Imaging. 2000;27(3):273-82.

4. Asnacios A, Courbon F, Rochaix P, Bauvin E, Cances-Lauwers V, Susini C, et al. Indium-111-pentetreotide scintigraphy and somatostatin receptor subtype 2 expression: new prognostic factors for malignant welldifferentiated endocrine tumors. J Clin Oncol. 2008;26(6):963-70.

5. Bombardieri E, Ambrosini V, Aktolun C, Baum RP, Bishof-Delaloye A, Del Vecchio S, et al. ${ }^{111}$ Inpentetreotide scintigraphy: procedure guidelines for tumour imaging. Eur J Nucl Med Mol Imaging. 2010;37(7):1441-8

6. Antunes P, Ginj M, Zhang H, Waser B, Baum RP, Reubi JC, et al. Are radiogallium-labelled DOTAconjugated somatostatin analogues superior to those labelled with other radiometals? Eur J Nucl Med Mol Imaging. 2007;34(7):982-93.

7. Bozkurt MF, Virgolini I, Balogova S, Beheshti M, Rubello D, Decristoforo C, et al. Guideline for PET/CT imaging of neuroendocrine neoplasms with ${ }^{68} \mathrm{Ga}$-DOTA-conjugated somatostatin receptor targeting peptides and ${ }^{18}$ F-DOPA. Eur J Nucl Med Mol Imaging. 2017;44(9):1588-601.

8. Du J, Arino-Estrada G, Bai X, Cherry SR. Performance comparison of dual-ended readout depth-encoding 
PET detectors based on BGO and LYSO crystals. Phys Med Biol. doi: 10.1088/1361-6560/abc365. Epub ahead of print. PMID: 33086214.

9. Badawi RD, Shi H, Hu P, Chen S, Xu T, Price PM, et al. First human imaging studies with the EXPLORER total-body PET scanner. J Nucl Med. 2019;60(3):299-303.

10. Tan H, Sui X, Yin H, Yu H, Gu Y, Chen S, et al. Total-body PET/CT using half-dose FDG and compared with conventional PET/CT using full-dose FDG in lung cancer. Eur J Nucl Med Mol Imaging. 2021;48(6):1966-75.

11. Liu G, Xu H, Hu P, Tan H, Zhang Y, Yu H, et al. Kinetic metrics of 18F-FDG in normal human organs identified by systematic dynamic total-body positron emission tomography. Eur J Nucl Med Mol Imaging. doi: 10.1007/s00259-020-05124-y. Epub ahead of print. PMID: 33416959.

12. Zhang YQ, Hu PC, Wu RZ, Gu YS, Chen SG, Yu HJ, et al. The image quality, lesion detectability, and acquisition time of 18F-FDG total-body PET/CT in oncological patients. Eur J Nucl Med Mol Imaging. 2020;47(11):2507-15.

13. Sandström M, Velikyan I, Garske-Román U, Sörensen J, Eriksson B, Granberg D, et al. Comparative biodistribution and radiation dosimetry of ${ }^{68} \mathrm{Ga}$-DOTATOC and ${ }^{68} \mathrm{Ga}$-DOTATATE in patients with neuroendocrine tumors. J Nucl Med. 2013;54(10):1755-9.

14. Woltering EA, Bergsland EK, Beyer DT. Neuroendocrine tumors of the stomach. American Joint Committee on Cancer 2017. In: Amin MB editor. AJCC Cancer Staging Manual. Eighth Edition. Springer 2017: 35159.

15. Tworowska I, Ranganathan D, Thamake S, Delpassand E, Mojtahedi A, Schultz MK, et al. Radiosynthesis of clinical doses of ${ }^{6}{ }^{8}$ Ga-DOTATATE (GalioMedix ${ }^{\mathrm{TM}}$ ) and validation of organic-matrix-based ${ }^{6}{ }^{8} \mathrm{Ge} /{ }^{6}{ }^{8}$ Ga generators. Nucl Med Biol. 2016;43(1):19-26. 
16. Gålne A, Almquist H, Almquist M, Hindorf C, Ohlsson T, Nordenström E, et al. A prospective observational study to eEvaluate the effects of long-acting somatostatin analogs on ${ }^{68} \mathrm{Ga}$-DOTATATE uptake in patients with neuroendocrine tumors. J Nucl Med. 2019;60(12):1717-23.

17. Ayati N, Lee ST, Zakavi R, Pathmaraj K, Al-Qatawna L, Poon A, et al. Long-acting somatostatin analog therapy differentially alters ${ }^{68} \mathrm{Ga}$-DOTATATE uptake in normal tissues compared with primary tumors and metastatic lesions. J Nucl Med. 2018;59(2):223-27.

18. Cox CPW, Segbers M, Graven LH, Brabander T, van Assema DME. Standardized image quality for 68GaDOTA-TATE PET/CT. EJNMMI Res.2020;10(1):27-36.

19. Nagaki A, Onoguchi M, Matsutomo N. Patient weight-based acquisition protocols to optimize (18)F-FDG PET/CT image quality. J Nucl Med Technol. 2011;39(2):72-6.

20. Hu P, Zhang Y, Yu H, Chen S, Tan H, Qi C, et al. Total-body 18F-FDG PET/CT scan in oncology patients: how fast could it be? Eur J Nucl Med Mol Imaging. doi: 10.1007/s00259-021-05357-5. Epub ahead of print. PMID: 33866409.

21. Ter Voert EEGW, Svirydenka H, Müller J, Becker AS, Balaz M, Efthymiou V, et al. Low-dose 18F-FDG TOF-PET/MR for accurate quantification of brown adipose tissue in healthy volunteers. EJNMMI Res. 2020;23;10(1):5-14.

22. Akamatsu G, Ishikawa K, Mitsumoto K, Taniguchi T, Ohya N, Baba S, et al. Improvement in PET/CT image quality with a combination of point-spread function and time-of-flight in relation to reconstruction parameters. J Nucl Med. 2012;53(11):1716-22. 


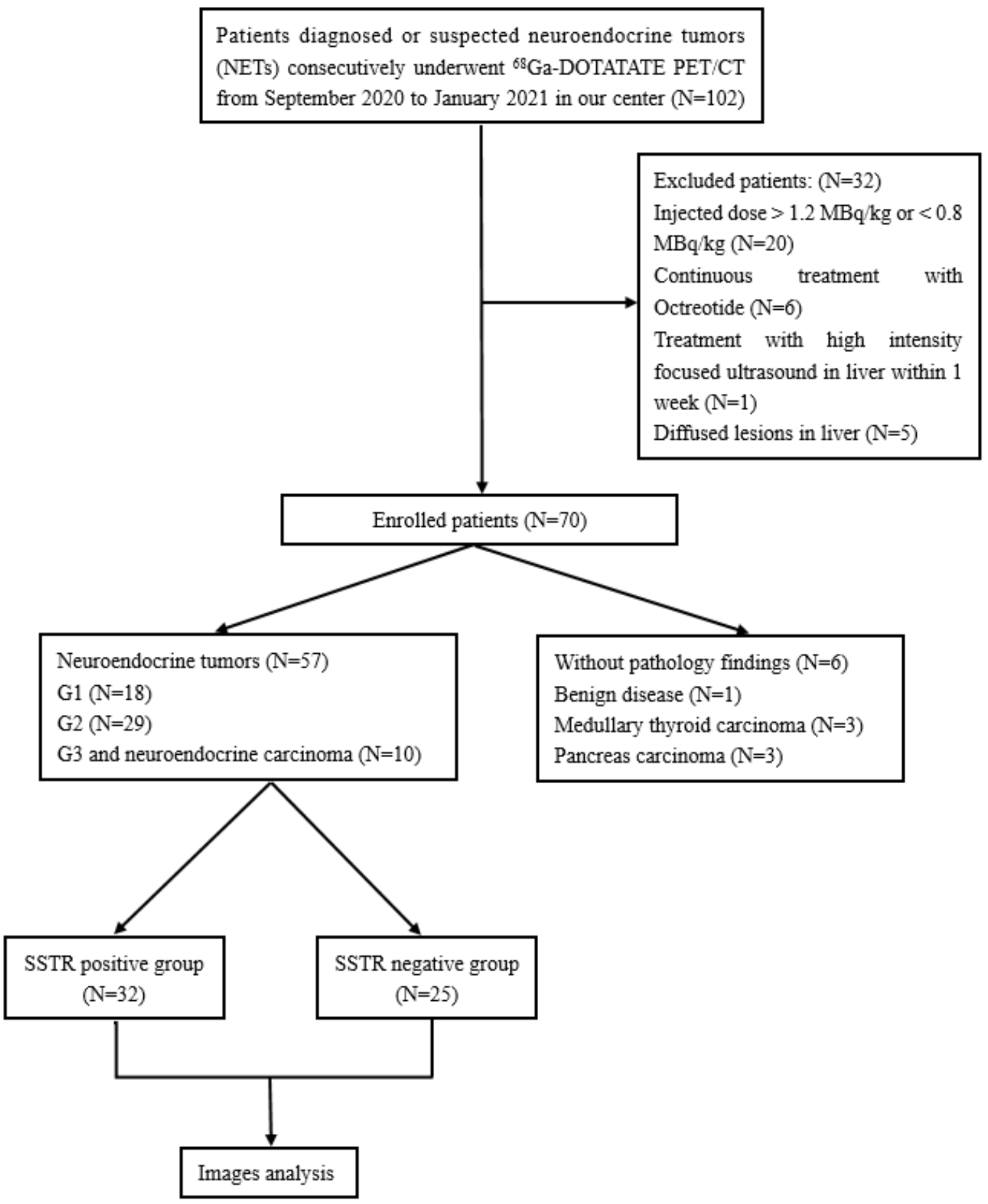

\section{Figure 1}

The Flowchart of the enrolled patients in our study. 


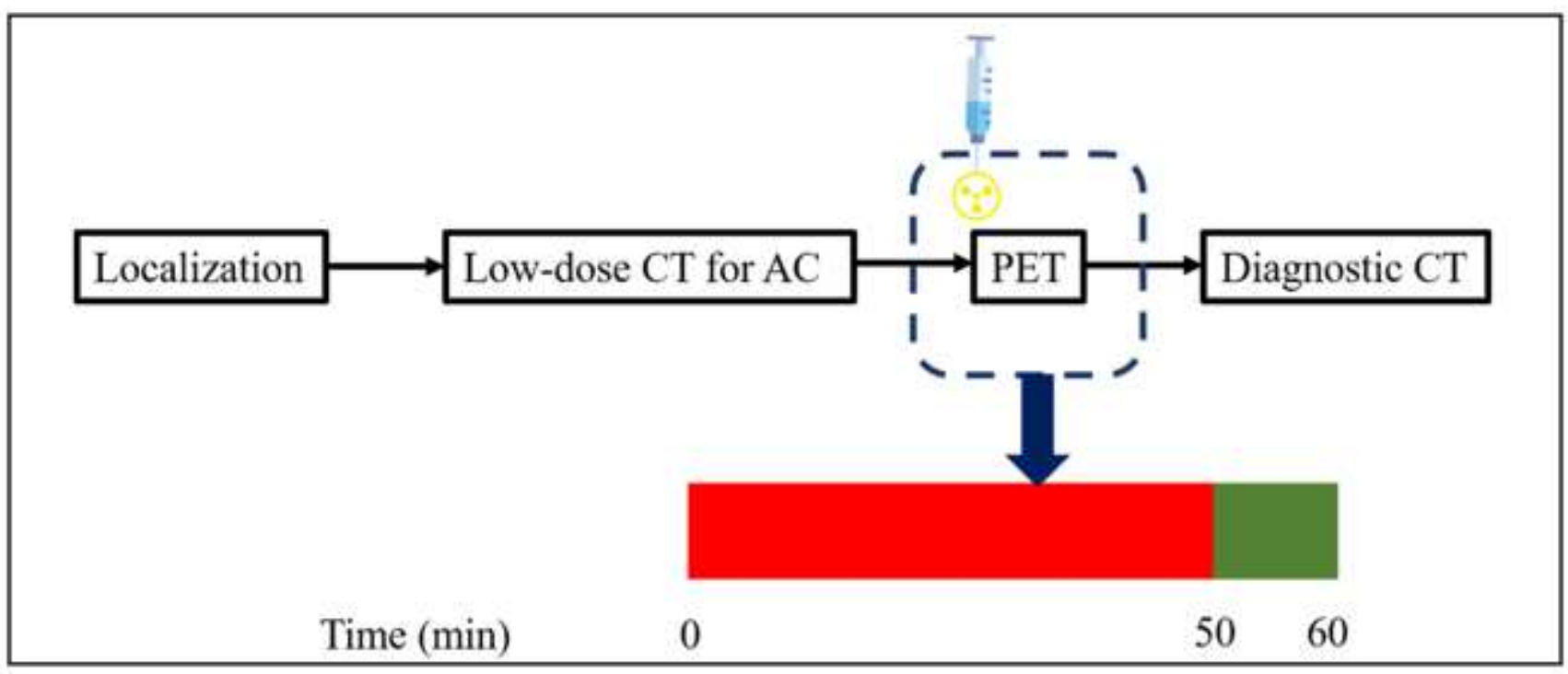

Figure 2

Diagram of basic acquisition protocol covering one bed position. $\mathrm{AC}=$ attenuation correction CT. 

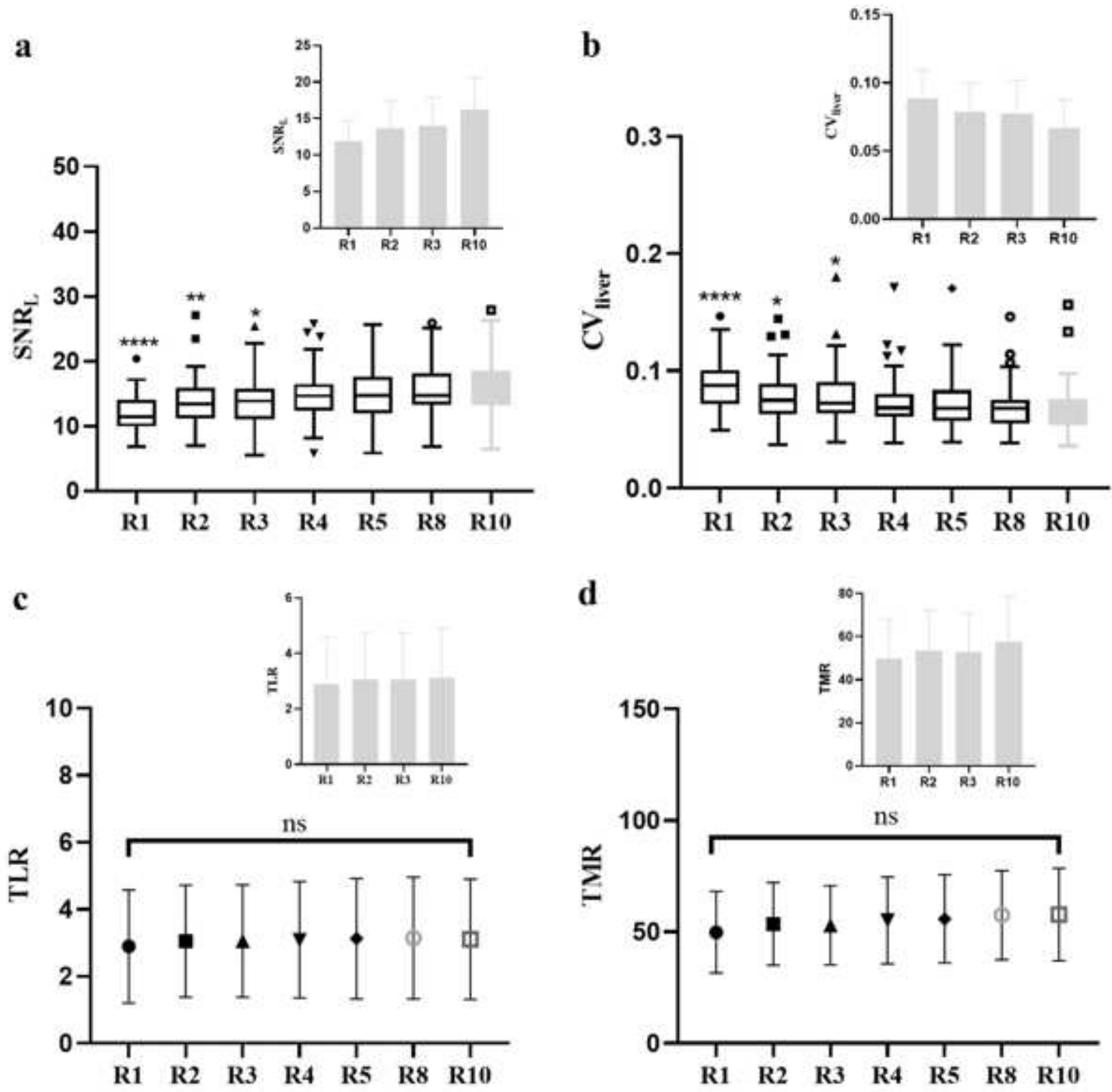

Figure 3

Comparasion of SNRL (a), CVliver (b), TLR (c) and TMR (d) between different acquisition groups. 


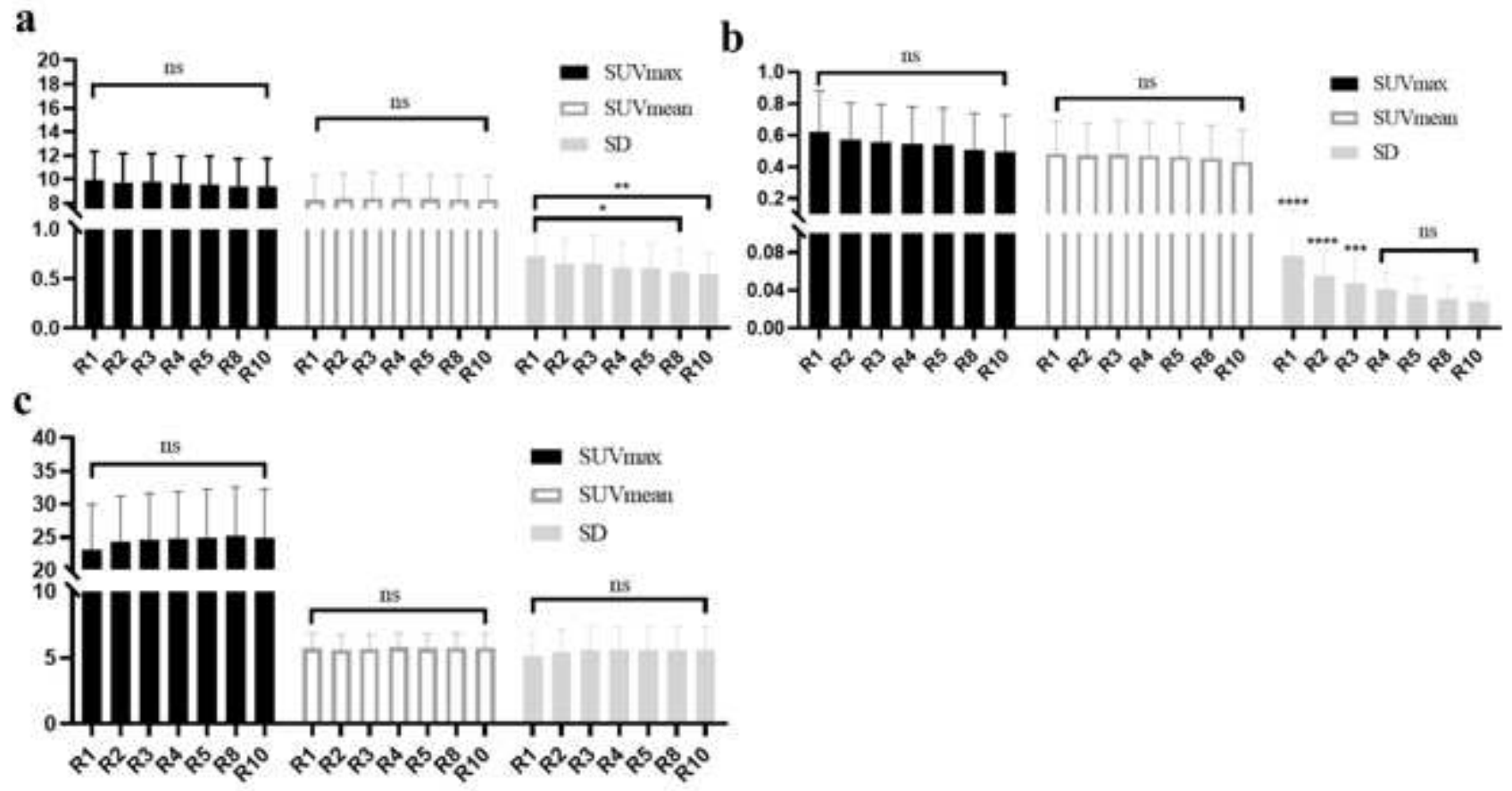

Figure 4

The SUVmax, SUVmean and SD of liver (a), blood pool (b) and lesions (c) between groups. 


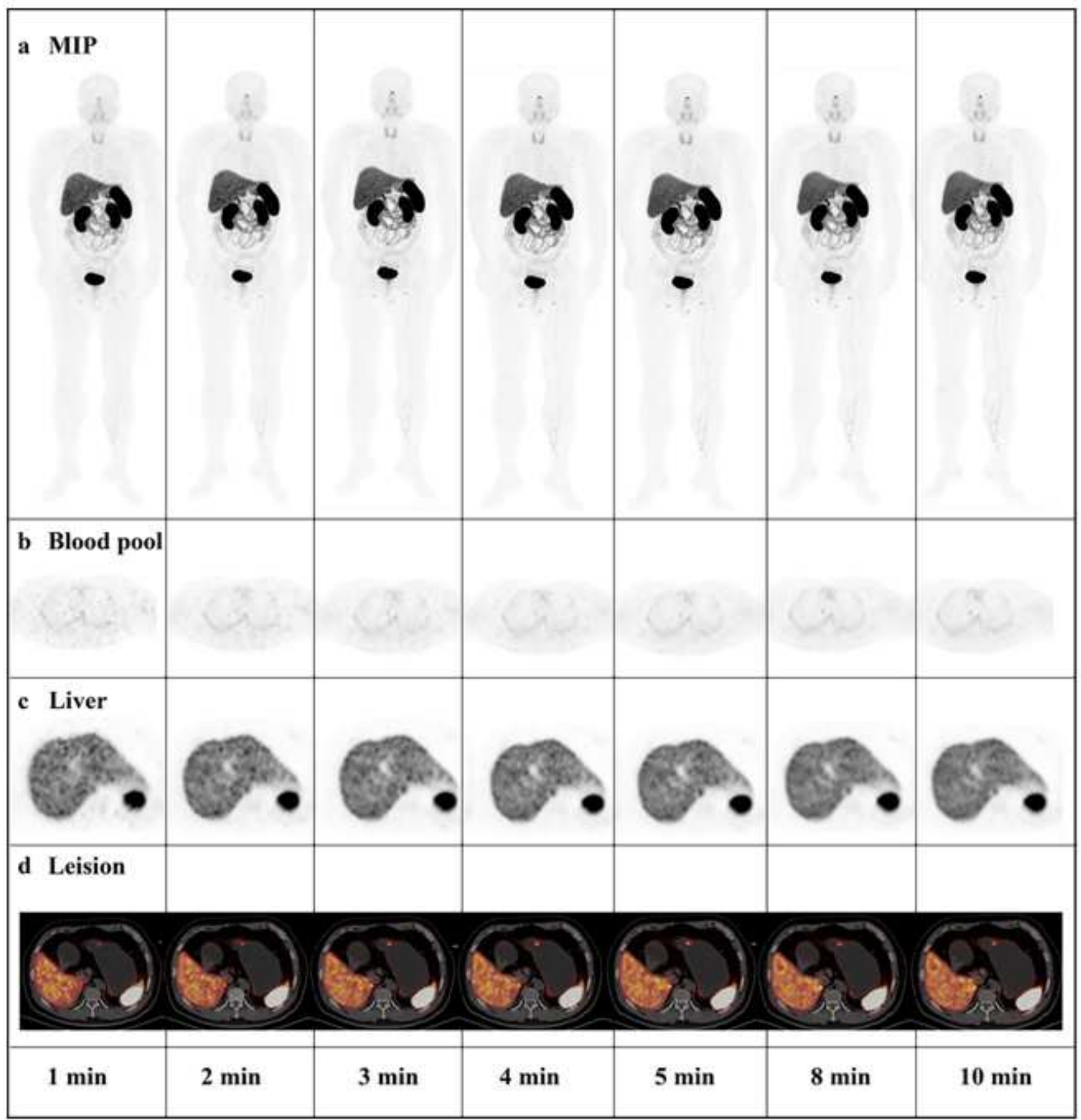

Figure 5

A-39-year-old man with G2 grade NET in gastric confirmed by gastroscope. Imaging of MIP (a) showed avid 68Ga-DOTATATE in abdominal region for R1-R10. The axial PET images showed corresponding mediastinal blood pool (b) and liver (c). The lesion in gastric body was evidently identified in all reconstruction times (c).

\section{Supplementary Files}


This is a list of supplementary files associated with this preprint. Click to download.

- Tables.pdf 